\title{
Network pharmacology-based active compounds and pharmacological mechanisms of ginseng for depression in post-COVID-19
}

\section{Na Wang}

shandong university of traditional chinese medicine

\section{Xianlei Wang}

National Oceanography Centre

Mengjiao He

shandong university of traditional chinese medicine

Wenxiu Zheng

shandong university of traditional chinese medicine

Xiaoqing Cai

Shandong University of traditional chinese medicine

Dongmei Qi

shandong university of traditional chinese medicine

\section{Yongqing Zhang}

shandong university of traditional chinese medicine

chunchao han ( $\sim$ chunchaoh@126.com )

shandong university of traditional chinese medicine

\section{Research}

Keywords: post-COVID-19, network pharmacology, ginseng, depression, immune-regulation

Posted Date: July 10th, 2020

DOI: https://doi.org/10.21203/rs.3.rs-40858/v1

License: (1) (1) This work is licensed under a Creative Commons Attribution 4.0 International License. Read Full License 


\section{Abstract}

Introduction: The novel coronavirus disease 2019 (COVID-19) is in the midst of worldwide panic. Sudden onset of an immediate life-threatening illness, quarantine and unemployment caused by epidemic are all contributors to depression. Ginseng has been reported to be an effective and safe clinical treatment on both immune-regulation and anti-depression. However, the mechanism of its anti-depression effect has not been fully characterized. In order to provide theoretical guidance for further clinical application in post-pandemic, we investigated active compounds and pharmacological mechanisms of ginseng to exert anti-depressant activity using network pharmacology, and discussed the active ingredients with immuneregulation and anti-depression.

Methods: Information on compounds in ginseng was obtained from public databases, and genes related to depression were gathered using the GeneCards database. Networks of ginseng-associated targets and depression-related genes were constructed through STRING database. Potential targets and pathway enrichment analysis related to the therapeutic efficacy of ginseng for depression were identified using Cytoscape and Database for Annotation, Visualization and Integrated Discovery (DAVID).

Results: Network pharmacological analysis of ginseng in treatment of depression identified 16 active ingredients, 47 potential targets, $32 \mathrm{GO}$ terms, and 8 target gene-regulated major pathways. Among them, kaempferol, beta-sitosterol, stigmasterol, fumarine and frutinone A are bioactive compounds and key chemicals. Core genes in PPI network were AKT1, CASP3, NOS3, TNF, and PPARG. Enrichment results revealed that ginseng could regulate multiple aspects of depression through neuroactive ligand-receptor interaction, HIF-1 signaling pathway, and Serotonergic synapse. More importantly, we found that frutinone $A$ and kaempferol are key ingredients in ginseng with dual activities of immune-regulation and anti-depression.

Conclusions: We discovered that the therapeutic activities of ginseng for depression mainly involve neurotransmitters, neurotrophic factors, neurogenesis, HPA axis and inflammatory response.

Pharmacological network analysis can help to explain the potential effects of ginseng for treating depression, indicating that ginseng is a preferable herb clinically for immune-regulation and antidepression in post-pandemic.

\section{Background}

The novel coronavirus disease 2019 (COVID-19), caused by the severe acute respiratory syndrome coronavirus-2, is in the midst of worldwide panic and global health concern since December 2019 [1]. The most common symptoms of COVID-19 illness are fever, cough, and dyspnea [2]. In the most severe cases, people with poor immunity may develop pneumonia and multiple organ failure, eventually leading to death. However, no specific drug or vaccine has yet been developed and the epidemic will not be brought under positive control in the short term. To our knowledge, the onset of a sudden and immediately lifethreatening illness could lead to posttraumatic stress disorder [3]. Quarantine is necessary to manage the 
outbreak, and the experience of being quarantined can, in some cases, lead to long-term adverse mental health consequences [4]. Furthermore, many other economic problems caused by epidemic, such as financial difficulties and loss of employment, undoubtedly increased public anxiety and depression. Based on these, it is of great significance to prevent the outbreak of depression after a global epidemic while improving the public immunity.

Panax ginseng Meyer, as a precious tonic traditional Chinese medicine (TCM), usually grows in cooler areas like Northeast China, Korea peninsula and Russia. It is known as the king of invigorating Qi and the Greek term "Panax", which means "cure of all diseases", implied its important position in the medical field. TCM theory emphasizes "Healthy Qi is stored inside, evil can not invade". Healthy Qi is equivalent to what we now call immunity. Ginseng can regulates each type of immune cells, therefore maintaining homeostasis of the immune system and enhancing resistance to illness or microbial attacks [5]. Quan et al. also found ginseng and salviae herbs play a role as immune activators and modulate immune responses during influenza virus infection [6]. As recorded in the traditional Chinese work, Jingyue Quanshu (Jing-yue's Complete Works), ginseng has been used in the classic prescription Qifu Yin to treat neurasthenia and other nervous system diseases. Ginsenosides, as the main pharmacologically active components of ginseng, have been found to exhibit as novel antidepressant agents [7]. It can be seen that ginseng has a good effect on both immune-regulation and anti-depression. Hao et al. integrated metabolomics and network pharmacology to study the immune-regulation mechanism of ginseng. Twenty compounds of ginseng associated with metabolomic changes were selected by the network pharmacology analysis, including ginsenoside Re, ginsenoside Rg1, frutinone A, and kaempferol [8]. However, its anti-depression therapeutic mechanisms have not yet been clearly elucidated.

Network pharmacology has been introduced in recent years for exploring the molecular mechanisms of TCM. The key ideas of emerging network pharmacology and network biology shares much with the holistic philosophy of TCM, updating the research paradigm from the current "one target, one drug" mode to a new "network target, multi-components" mode [9]. Through bridging the emerging network science and ancient TCM, we obtain novel methodologies and opportunities for discovering bioactive ingredients and biomarkers, potentially revealing mechanisms of action. Even though the anti-depression effects of ginseng have been reported, network pharmacology-based prediction of the bioactive components and target pathways has not been performed.

In this study, we investigated active compounds and pharmacological mechanisms of ginseng to exert anti-depressant activity using network pharmacology. The fowchart of the experimental procedures of our study is shown in Fig. 1. We also discussed the active ingredients with immune-regulation and antidepression, in order to provide theoretical guidance for further clinical application in post-pandemic.

\section{Methods}

\subsection{Database construction and ADME screening of ginseng ingredients}


All of the known ingredients of ginseng were manually collected from related literature and phytochemical databases [10]: Traditional Chinese Medicine Systems Pharmacology Database (TCMSP, http://tcmspw.com/tcmsp.php). An in silico integrative model ADME (absorption, distribution, metabolism, and excretion) was used to select the ingredients with favorable pharmacokinetics properties. The parameter used in this study included oral bioavailability (OB) and drug-likeness (DL). OB refers to the rate and degree of drug absorption into human circulation after oral administration and DL refers to the similarity of a compound to a known drug. They are two of the most commonly used pharmacokinetic properties in drug screening. Ingredients conforming to the requirements of both $\mathrm{OB}$ $\geq 30 \%$ and $\mathrm{DL} \geq 0.18$ were regarded as active ingredients in ginseng for further analysis.

\subsection{Target genes related to the identified compounds}

In the TCMSP database, the potantial targets of each active ingredient were found. The Uniprot data library (https://www.uniprot.org/) was used to convert the selected target into a gene name of Homo sapiens (Human) to prepare the target genes related to the identified compounds. Component-target data of ginseng were obtained after deleting the duplicates.

\subsection{Collection of therapeutic targets of ginseng for depression}

Information on depression-associated target genes was collected from the human gene database (GeneCards, http://www.genecards.org/) using "depression" as the keywords, and only "Homo sapiens" proteins linked to depression were selected. With relevance score $>7.32$ as the threshold, the obtained targets were screened. In order to obtain the potential anti-depressant targets of ginseng, the targets of each active ingredient were intersected with depression-related targets through an online mapping platform (http: //bioinfo. Genotoul.fr/jvenn/example.html).

\subsection{Protein-protein interaction data}

The potential anti-depressant targets of ginseng were imported into the String database (http://stringdb.org/) for the analysis of protein-protein interaction (PPI). A confidence score above 0.4 indicates an interaction relationship. So the confidence was set as confidence $>0.4$, while other parameters remained defaults.

\subsection{Construction of the pharmacological networks}

All visualized network models were established via Cytoscape 3.8.0 (http://www.cytoscape.org/), an open software package project for visualizing, integrating, modeling and analyzing the interaction networks [11]. In the network, nodes represent compounds or target genes, while edges represent the interactions between them. The "Network Analyze" in Cytoscape 3.8.0 is used to analyze the topology properties of the Network. Three networks were constructed: (1) Compound-compound target network. The potential targets of each active ingredient in ginseng were used to generate it; (2) Compound-target-disease network. The potential anti-depressant targets of ginseng were used to generate it; (3) PPI network of depression-related targets in ginseng. 


\subsection{Gene ontology and pathway enrichment analysis}

The potential anti-depressant targets of ginseng was input into the Database for Annotation, Visualization and Integrated Discovery (DAVID, https://david.ncifcrf.gov/tools.jsp) for Gene Ontology (GO) and Kyoto Encyclopedia of Genes and Genomes (KEGG) pathway enrichment analysis. Gene species and background were limited to human. The false discovery rate $(F D R)<0.05$ was used as the filter to obtain the top $\mathrm{GO}$ terms of biological process (BP), cell component (CC) and molecular function (MF). The enrichment analysis of KEGG metabolic pathway was screened under the conditions of $\mathrm{P}<$ 0.01 and FDR $<0.05$. The results were visualized using Graphpad Prism 8.0.2 (https://www.graphpad.com/).

\section{Results}

\subsection{Candidate compound screening for ginseng}

Retrieved from TCMSP, there were 190 components related to ginseng in total. Under the screening thresholds of $O B \geq 30 \%$ and $D L \geq 0.18,22$ active ingredients were selected for further analysis (Table 1 ). Among them, there are five active ingredients with higher OB: Celabenzine (101.88\%), Aposiopolamine (66.65\%), Frutinone A (65.9\%), Inermin (65.83\%) and Girinimbin (61.22\%). These components play important roles in the pharmacological activities of ginseng.

\subsection{Potential target prediction for ginseng}

Among the 22 active components, 5 of them did not find corresponding targets, while the remaining 17 components obtained 109 potential targets after removing duplicates. All active compounds, their targets and the interactions between them are presented in the compound-compound target network (Fig. 2), which is composed of 126 nodes ( 17 components and 109 targets) and 246 edges. The pink nodes represent drug targets while the blue nodes represent compounds, and the edges represent the interactions between them. Degree, a topological parameter describing the importance of a node, stands for the number of edges connecting to the node. We used it to further determine the importance of each active component. Through analysis, we found that RS5 exhibited the largest number of potential targets connections (Kaempferol, degree = 59), followed by RS3 (Beta-sitosterol, degree $=36$ ), RS2 (Stigmasterol, degree = 31), RS17 (Fumarine, degree = 24) and RS4 (Inermin, degree = 16). The same active ingredient can act on different targets, and different active ingredients can also act on the same target, which fully reflects the multi-component, multi-target action characteristics of ginseng.

\subsection{Collection of therapeutic targets of ginseng for depression}

A total of 11478 disease targets were obtained from GeneCards with "depression" as the keyword. Among them, 975 depression-related genes meet the requirement of relevance score $>7.32$ and ginseng have 47 potential targets for depression (Fig. 3a). Compound-target-disease network (Fig. 3b) with 63 nodes and 102 edges linked 16 compounds and 47 target genes related to depression. Among the 16 candidate 
compounds, RS5 exhibited the largest number of potential anti-depression targets connections (Kaempferol, degree $=24)$, followed by RS3 (Beta-sitosterol, degree $=18)$, RS2 (Stigmasterol, degree $=14$ ), RS17 (Fumarine, degree $=9$ ) and RS10 (Frutinone A, degree $=8$ ). For the 47 potential anti-depression targets, the network showed PTGS2 had the largest number of compound-target interactions, followed by SCN5A, GABRA1, CHRNA7 and SLC6A4, while the remaining 42 targets showed interactions with up to three compounds.

\subsection{Protein-protein interaction analysis}

After removing a free target, the PPI network (Fig. 4) contains 46 nodes and 259 edges, with average node degree of 11 and average local clustering coefficient of 0.533 . Target size and color are used to reflect the degree, while edge thickness and color are used to reflect the combine score. The important targets were painted red and located centrally in the network. AKT1 (degree = 25), CASP3 (degree = 20), NOS3 (degree = 19), TNF (degree = 19), PPARG (degree = 18), SLC6A4 (degree = 18), ACHE (degree = 18), IL1B (degree = 17), PTGS2 (degree $=17$ ) and MAOA (degree $=16)$ were the top ten genes regarding their degree. AKT1 also has the highest closeness centrality (0.67), indicating the faster the signal is transferred to other nodes. Due to the highest betweenness centrality (0.14), AKT1 is considered a bottleneck node in monopolistic position between modules in the network and more suitable as a therapeutic target.

\subsection{GO biological process and KEGG pathway enrichment analysis}

Through DAVID database, we obtained $305 \mathrm{GO}$ terms (226 BP terms, $31 \mathrm{CC}$ terms and $48 \mathrm{MF}$ terms) and 68 pathways in total. With FDR $<0.05$ as the screening condition, $32 \mathrm{GO}$ terms ( $22 \mathrm{BP}$ terms, $6 \mathrm{CC}$ terms and $4 \mathrm{MF}$ terms) were selected (Fig. 5). GO analysis revealed that target genes were majorly associated with the biological process of response to drug (GO:0042493), positive regulation of cell proliferation (GO:0008284), positive regulation of nitric oxide biosynthetic process (G0:0045429), response to hypoxia (G0:0001666), positive regulation of ERK1 and ERK2 cascade (G0:0070374), cellular response to organic cyclic compound (G0:0071407), response to nicotine (G0:0035094), regulation of insulin secretion (G0:0050796), cellular response to hypoxia (G0:0071456), response to ethanol (G0:0045471) and etc. The 6 cell component terms were involved in plasma membrane (G0:0005886), integral component of plasma membrane (G0:0005887), membrane raft (GO:0045121), neuron projection (GO:0043005), postsynaptic membrane (G0:0045211) and Caveola (G0:0005901). Among them, plasma membrane and integral component of plasma membrane accounted for the largest proportion, with 29 and 15 targets respectively. Depending on the outcomes of GO enrichment, the enriched molecular function ontologies were dominated by protein homodimerization activity (GO:0042803), enzyme binding (G0:0019899), heme binding (GO:0020037), extracellular ligand-gated ion channel activity (GO:0005230). Under the conditions of $\mathrm{P}<0.01$ and FDR $<0.05$ (Table 2), we found that pathways in neuroactive ligand-receptor interaction (hsa04080), African trypanosomiasis (hsa05143), HIF-1 signaling pathway (hsa04066), Leishmaniasis (hsa05140), Toxoplasmosis (hsa05145), Serotonergic synapse (hsa04726), Tuberculosis (hsa05152) and Malaria (hsa05144) are the interaction pathways that ginseng exerts combined antidepressant effects (Fig. 6). 


\section{Discussion}

Depression is an complex disorder with multiple etiologies including genetic, epigenetic, and environmental factors. Some possible pathophysiological mechanisms of depression include altered neurotransmission, hypothalamic-pituitary-adrenal (HPA) axis abnormalities involved in chronic stress, inflammation, reduced neuroplasticity, and network dysfunction. All of the proposed mechanisms are not separate but integrally related and interact bidirectionally [12]. Currently, many antidepressants work on a single target. Selective serotonin reuptake inhibitors (SSRIs) have been the first line of antidepressants for the past 25 years, although they have only moderate efficacy and may take weeks to produce measurable benefits. TCM is characterized by multi-target, multi-pathway and low side effect. Multi-component therapy produces "synergies", in which the combined effects are greater than the sum of the individual effects. To our knowledge, the anti-depression mechanisms of active ingredients from TCM can be summed up as following: increasing synaptic availability of monoamines [13], alleviation of the HPA axis dysfunctions [14], regulation of the cAMP signaling pathway [15] and caspases [16], and amelioration of the dysregulation of immune and inflammation [17]. Ginseng has been shown to be a precious herbal medicine with dual activities of immune-regulation and anti-depression. In this study, we investigated active compounds and pharmacological mechanisms of ginseng to exert anti-depressant activity using network pharmacology.

The screened active components can be classified as flavonoids, saponins, sterols, organic acids and etc. It is not difficult to find that some nodes are more concentrated in the network than others. That is, the compound-compound target space was biased towards certain compounds and targets [18]. As the most common flavonoid, kaempferol has attracted much attention due to its anti-cancer, anti-inflammatory, antioxidant, antibacterial and antiviral effects. Gao et al. have comfirmed that antidepressive effects of kaempferol are mediated by reduction of oxidative stress, proinflammatory cytokines and up-regulation of AKT/ $\beta$-catenin cascade [19]. Stigmasterol has been found to exert neuro-protective effect through reduction of oxidative stress and inactivation of autophagy via AMPK/mTOR and JNK pathways [20]. It has been reported that $\beta$-sitosterol has protective effects on various brain-related diseases independent of their lipid-lowering effects. $\beta$-sitosterol can also suppress the development of cerebral aneurysm by inhibiting inflammatory reactions including TNF-a [21]. This indicated that our prediction of the active ingredient in ginseng that plays an antidepressant role is reasonable. However, frutinone A are mostly considered as an active ingredient of the broad spectrum antimicrobial herbal extract up to now and antidepression may be its potential pharmacological activity that has not yet been discovered. The compound-target-disease network showed intimate communications between several components and multiple targets, and facilitated a better understanding of the possible curative mechanism of ginseng in the treatment of depression.

In the PPI network, AKT1 has the highest degree, closeness centrality and betweenness centrality. It is in the monopolistic position between modules in the network and more suitable as a therapeutic target. AKT1, a downstream enzyme that has been implicated in the pathogenesis of serotonin-related disorders, facilitates growth factor-mediated cell survival and block apoptosis through the regulation of its 
downstream effector (GSK-3) in depressants [22-23]. Thus, the regulation of AKT1 activity by ginseng (kaempferol) inhibit and alleviate depression by a variety of ways. Moreover, SLC6A4 is responsible for the serotonergic neurotransmission, which is an important substance that regulates neural activity. The serotonin-linked polymorphic region (5-HTTLPR) is a degenerate repetition of the gene encoding the 5hydroxytryptamine transporter (SLC6A4). The S/S genotype in this region is associated with decreased serotonin expression and increased susceptibility to depression [12]. TNF and IL-1B may be involved in major depression not just by activating certain cascades of inflammation but by many other mechanisms [24]. It is not difficult to see that ginseng's treatment of depression involves neurotransmitters (especially serotonin), inflammation, neurotrophic factors and many other aspects. Furthermore, inflammatory cytokines have been shown to reduce monoamine levels in depressed patients by increasing the metabolism of tryptophan, an important precursor to serotonin [12]. It also reconfirmed the factors that influence depression are not separate but integrally related and interact bidirectionally. These results were consistent with our enrichment analysis, and have been previously reported to be related to depression.

GO analysis revealed that target genes were majorly associated with the biological process of response to drug, indicated the key requirement of avoiding drug dependence in depression drug development and clinical treatment [25]. Through positive regulation of cell proliferation, ginseng affect neurogenesis, which is thought to contribute to anti-stress recovery and underlie the clinical efficacy of antidepressants [26]. Extracellular signal-regulated kinase $1 / 2$ (ERK1/2) signaling, which belongs to the large family of mitogen-activated protein kinase signaling cascases, showed low activity in the frontal cortex (Brodmann $8,9,10)$ and hippocampus in depressed patients [27]. It has been shown to have significant effects on the regulation of long-term enhancement, long-term depression, and neuronal survival through neurotrophic/growth factors [28-29]. In recent years, more and more studies have proved that astrocytes not only have a supporting effect, but also regulate physiological processes by secreting neurotrophic factors. This suggests that ginseng can play a direct and indirect therapeutic role by acting on astrocytes or nerve cells. Glucocorticoid is the main cytokine released by macrophages, and ginseng's effect on lipopolysaccharide-mediated signaling pathway is important to regulate inflammation and HPA axis dysfunction. The main cell component terms were involved in plasma membrane and integral component of plasma membrane, while the enriched molecular function ontologies were dominated by protein homodimerization activity and enzyme binding. In addition, KEGG pathway analysis were primarily pertaining to neuroactive ligand-receptor interaction, serotonergic synapse and HIF-1 signaling pathway, which followed the previous reports that these pathways participate in crucial functions in the progression and development of depression. It is worth mentioning that there were several pathways which are concerned with diseases, such as African trypanosomiasis, Leishmaniasis, Toxoplasmosis, Tuberculosis and Malaria, prompting that ginseng may have potential therapeutic efects on these diseases. Twelve targets enriched onto neuroactive ligand-receptor interaction pathway were mostly neurotransmitter receptors. Monoamine neurotransmitters (serotonin, norepinephrine and dopamine) have a potential role in the pathogenesis of depression. The monoamine hypothesis explained how antidepressants work [30]. HIF-1 signaling pathway is associated with AKT, NF-KB signaling pathways and the transcription of VEGF, which interferes with inflammatory injury, oxidative stress, apoptosis and 
tumor growth. It is FG-4592 that improves depressive-like behaviors through HIF-1-mediated neurogenesis and synapse plasticity in rats [31]. All of the proposed biological pathophysiological mechanisms of depression are reciprocally connected with each other. For example, inflammation leads to HPA hyperactivity, which in turn plays a permissive role in the inflammatory process, forming a vicious circle. Through enrichment analysis, we discovered that the therapeutic activities of ginseng for depression involve neurotransmitters, neurotrophic factors, neurogenesis, HPA axis and inflammatory response. Taken together, these results indicate that ginseng can regulate whole-body systems through a complex genes-interaction network, resulting in a certain effect in depression.

As mentioned above, ginsenoside Re, ginsenoside Rg1, frutinone A and kaempferol were the key ingredients in ginseng for immune-regulation, regulating the choline metabolism through acetylcholinesterase or cholinesterase [32]. Through analysis, we found frutinone A and kaempferol are also the major ingredients in ginseng to exert anti-depressant activity. Therefore, these two ingredients are the key ingredients in ginseng with dual activities of immune-regulation and anti-depression. These findings could provide theoretical guidance in the investigation of ginseng for further clinical application in post-COVID-19. However, experiment is still needed to validate the interactions between drugs and proteins based on theoretical predictions.

\section{Conclusions}

Past experience shows that mental health problems often occur in the public following a major epidemic situation. In this study, we investigated the active compounds and pharmacological mechanisms of ginseng to exert anti-depressant activity using network pharmacology, and discussed the active ingredients with immune-regulation and anti-depression. Kaempferol, beta-sitosterol, stigmasterol, fumarine and frutinone $A$ are major ingredients in ginseng to exert anti-depressant activity. We discovered that the therapeutic activities of ginseng for depression mainly involve neurotransmitters, neurotrophic factors, neurogenesis, HPA axis and inflammatory response. A total of 47 potential targets were identified, which were significantly enriched in the neuroactive ligand-receptor interaction, serotonergic synapse and HIF-1 signaling pathway, indicating that the therapeutic effects of ginseng were produced by synergistic complex interactions among its chemical compounds and related targets. More importantly, frutinone A and kaempferol are key ingredients in ginseng with dual activities of immune-regulation and antidepression.

These findings could be guidelines in the investigation of ginseng to exert anti-depressant activity, in order to provide theoretical guidance for further clinical application in post-COVID-19.

\section{Abbreviations}

COVID-19, coronavirus disease 2019; TCM, traditional Chinese medicine; OB, oral bioavailability; DL, druglikeness; TCMSP, Traditional Chinese Medicine Systems Pharmacology Database; ADME, absorption, distribution, metabolism, and excretion; DAVID, Database for Annotation, Visualization and Integrated 
Discovery; PPI, protein-protein interaction; HPA axis, hypothalamic-pituitary-adrenal axis; GO, Gene Ontology; KEGG, Kyoto Encyclopedia of Genes and Genomes; FDR, false discovery rate; BP, biological process; CC, cell component; MF, molecular function.

\section{Declarations}

\section{Author contributions}

NW wrote the paper and drew the figures; XLW sorted the data and created the tables; MJH and WXZ modified the tables and revised the paper; MQD and XQC revised the paper; $Y Q Z$ and $C C H$ directed the research and revised the paper.

\section{Acknowledgements}

Not applicable.

\section{Competing interests}

The authors declare no competing interests.

\section{Availability of data and materials}

The data and materials generated or analyzed during this study are available from the corresponding author on reasonable request.

\section{Consent for publication}

The manuscript is approved by all authors for publication.

\section{Funding}

This work was supported by the Project of Shandong Province Key Research and Development Program [Grant No. 2017YYSP030]. Traditional Chinese Medicine Resources and Utilization Innovation Team. Shandong Provincial Natural Science Foundation, China (No. ZR2017MH086). Major Science and Technology Innovation in Shandong Province [Grant No. 2017CXGC1307]. Key Laboratory of Classical Theory of Traditional Chinese Medicine, Ministry of Education. Shandong Provincial Key Laboratory of Traditional Chinese Medicine for Basic research.

\section{References}

1. World Health Organization (WHO), 2020a. Laboratory testing of 2019 novel coronavirus (2019-nCoV) in suspected human cases. https://apps.who.int/iris/handle/10665/ 330676

2. Asadi-Pooya AA, Simani L. Central nervous system manifestations of COVID-19: A systematic review. J Neurol Sci. 2020;413:116832. 
3. Wu KK, Chan SK, Ma TM. Posttraumatic stress, anxiety, and depression in survivors of severe acute respiratory syndrome (SARS). J Trauma Stress. 2005;18(1):39-42.

4. Liu X, Kakade M, Fuller CJ, Fan, B., Fang, Y., Kong, J., et al. Depression after exposure to stressful events: lessons learned from the severe acute respiratory syndrome epidemic. Compr Psychiatry. 2012;53(1):15-23.

5. Kang S, Min H. Ginseng, the 'Immunity Boost': The Effects of Panax ginseng on Immune System. J Ginseng Res. 2012;36(4):354-368.

6. Quan FS, Compans RW, Cho YK, Kang SM. Ginseng and Salviae herbs play a role as immune activators and modulate immune responses during influenza virus infection. Vaccine. 2007;25(2):272-282.

7. Wang YS, Shen CY, Jiang JG. Antidepressant active ingredients from herbs and nutraceuticals used in TCM: pharmacological mechanisms and prospects for drug discovery. Pharmacol Res. 2019;150:104520.

8. Hao J, Hu H, Liu J, Wang, X., Liu, X., Wang, J., et al. Integrated Metabolomics and Network Pharmacology Study on Immunoregulation Mechanisms of Panax ginseng through Macrophages. Evid Based Complement Alternat Med. 2019;2019:3630260.

9. Li S, Zhang B. Traditional Chinese medicine network pharmacology: theory, methodology and application. Chin J Nat Med. 2013;11(2):110-120.

10. Jinlong Ru; Peng Li; Jinan Wang; Wei Zhou; Bohui Li; Chao Huang; Pidong Li; Zihu Guo; Weiyang Tao; Yinfeng Yang; Xue Xu; Yan Li; Yonghua Wang; Ling Yang. TCMSP: a database of systems pharmacology for drug discovery from herbal medicines. J Cheminformatics. 2014 Apr 16;6(1):13.

11. Li L, Qiu H, Liu M, Cai Y. A Network Pharmacology-Based Study of the Molecular Mechanisms of Shaoyao-Gancao Decoction in Treating Parkinson's Disease. Interdiscip Sci. 2020;12(2):131-144.

12. Dean J, Keshavan M. The neurobiology of depression: An integrated view. Asian J Psychiatr. 2017;27:101-111. doi:10.1016/j.ajp.2017.01.025

13. Delgado PL. Depression: the case for a monoamine deficiency. J Clin Psychiatry. 2000;61 Suppl 6:711.

14. Ott V, Friedrich M, Prilop S, Lehnert, H., Jauch-Chara, K., Born, J., et al. Food anticipation and subsequent food withdrawal increase serum cortisol in healthy men. Physiol Behav. 2011;103(5):594-599. doi:10.1016/j.physbeh.2011.04.020

15. Castrén E, Rantamäki T. The role of BDNF and its receptors in depression and antidepressant drug action: Reactivation of developmental plasticity. Dev Neurobiol. 2010;70(5):289-297. doi:10.1002/dneu.20758

16. Coyle JT, Duman RS. Finding the intracellular signaling pathways affected by mood disorder treatments. Neuron. 2003;38(2):157-160. doi:10.1016/s0896-6273(03)00195-8

17. Shalini S, Dorstyn L, Dawar S, Kumar S. Old, new and emerging functions of caspases. Cell Death Differ. 2015;22(4):526-539. 
18. Hong M, Zhang Y, Li S, Tan, H. Y., Wang, N., Mu, S., et al. A Network Pharmacology-Based Study on the Hepatoprotective Effect of Fructus Schisandrae. Molecules. 2017;22(10):1617.

19. Gao W, Wang W, Peng Y, Deng Z. Antidepressive effects of kaempferol mediated by reduction of oxidative stress, proinflammatory cytokines and up-regulation of AKT/ $\beta$-catenin cascade. Metab Brain Dis. 2019;34(2):485-494.

20. Sun J, Li X, Liu J, Pan X, Zhao Q. Stigmasterol Exerts Neuro-Protective Effect Against Ischemic/Reperfusion Injury Through Reduction Of Oxidative Stress And Inactivation Of Autophagy. Neuropsychiatr Dis Treat. 2019;15:2991-3001. Published 2019 Oct 18.

21. Yang Q, Yu D, Zhang Y. $\beta$-Sitosterol Attenuates the Intracranial Aneurysm Growth by Suppressing TNF-a-Mediated Mechanism. Pharmacology. 2019;104(5-6):303-311.

22. Pereira PA, Bicalho MA, de Moraes EN, et al. Genetic variant of AKT1 and AKTIP associated with lateonset depression in a Brazilian population. Int J Geriatr Psychiatry. 2014;29(4):399-405.

23. Wang YS, Shen CY, Jiang JG. Antidepressant active ingredients from herbs and nutraceuticals used in TCM: pharmacological mechanisms and prospects for drug discovery. Pharmacol Res. 2019;150:104520.

24. Schmidt FM, Koch J, Nowak C, Holdt, L. M., Teupser, D., Hegerl, U., et al. Ligands and receptors of the TNF superfamily are decreased in major depression and during early antidepressant therapy. $J$ Psychiatr Res. 2019;119:116-121.

25. Liu Y, Fan P, Zhang S, Wang Y, Liu D. Prioritization and comprehensive analysis of genes related to major depressive disorder. Mol Genet Genomic Med. 2019;7(6):e659.

26. Kraus C, Castrén E, Kasper S, Lanzenberger R. Serotonin and neuroplasticity-links between molecular, functional and structural pathophysiology in depression. Neurosci Biobehav Rev 2017; 77 : 317-26.

27. Dwivedi Y, Rizavi HS, Roberts RC, Conley RC, Tamminga CA, Pandey GN. Reduced activation and expression of ERK1/2 MAP kinase in the post-mortem brain of depressed suicide subjects. J Neurochem. 2001;77(3):916-928.

28. Dwivedi Y, Rizavi HS, Zhang H, Roberts RC, Conley RR, Pandey GN. Aberrant extracellular signalregulated kinase (ERK)1/2 signalling in suicide brain: role of ERK kinase 1 (MEK1). Int J Neuropsychopharmacol. 2009;12(10):1337-1354.

29. Giovannini MG. The role of the extracellular signal-regulated kinase pathway in memory encoding. Rev Neurosci. 2006;17(6):619-634.

30. Numakawa T, Suzuki S, Kumamaru E, Adachi N, Richards M, Kunugi H. BDNF function and intracellular signaling in neurons. Histol Histopathol. 2010;25(2):237-258.

31. Malhi GS, Mann JJ. Depression. Lancet. 2018;392(10161):2299-2312.

Li G, Zhao M, Cheng X, Zhao, T., Feng, Z., Zhao, Y., et al. FG-4592 Improves Depressive-Like Behaviors through HIF-1-Mediated Neurogenesis and Synapse Plasticity in Rats. Neurotherapeutics. 2020;17(2):664675. 


\section{Tables}

Table 1 Active compounds and ADME parameters of ginseng ( $\mathrm{OB} \geq 30 \%$, $\mathrm{DL} \geq 0.18$ ). 


\begin{tabular}{|c|c|c|c|c|}
\hline ID & MOL ID & Molecule Name & $\mathrm{OB}(\%)$ & DL \\
\hline RS1 & MOL002879 & Diop & 43.59 & 0.39 \\
\hline RS2 & MOL000449 & Stigmasterol & 43.83 & 0.76 \\
\hline RS3 & MOL000358 & Beta-sitosterol & 36.91 & 0.75 \\
\hline RS4 & MOL003648 & Inermin & 65.83 & 0.54 \\
\hline RS5 & MOL000422 & Kaempferol & 41.88 & 0.24 \\
\hline RS6 & MOL005308 & Aposiopolamine & 66.65 & 0.22 \\
\hline RS7 & MOL005317 & Deoxyharringtonine & 39.27 & 0.81 \\
\hline RS8 & MOL005318 & Dianthramine & 40.45 & 0.20 \\
\hline RS9 & MOL005320 & Arachidonate & 45.57 & 0.20 \\
\hline RS10 & MOL005321 & Frutinone A & 65.9 & 0.34 \\
\hline RS11 & MOL005344 & Ginsenoside Rh2 & 36.32 & 0.56 \\
\hline RS12 & MOL005348 & Ginsenoside Rh4 & 31.11 & 0.78 \\
\hline RS13 & MOL005356 & Girinimbin & 61.22 & 0.31 \\
\hline RS14 & MOL005376 & Panaxadiol & 33.09 & 0.79 \\
\hline RS15 & MOL005384 & Suchilactone & 57.52 & 0.56 \\
\hline RS16 & MOL005399 & Alexandrin & 36.91 & 0.75 \\
\hline RS17 & MOL000787 & Fumarine & 59.26 & 0.83 \\
\hline RS18 & MOL004492 & Chrysanthemaxanthin & 38.72 & 0.58 \\
\hline RS19 & MOL005314 & Celabenzine & 101.88 & 0.49 \\
\hline RS20 & MOL005357 & Gomisin B & 31.99 & 0.83 \\
\hline
\end{tabular}




$\begin{array}{ccccc}\text { RS21 } & \text { MOL005360 } & \text { Malkangunin } & 57.71 & 0.63 \\ \text { RS22 } & \text { MOL005401 } & \text { Ginsenoside Rg5 } & 39.56 & 0.79\end{array}$

Table 2 Functions of potential target genes based on KEGG pathway analysis.

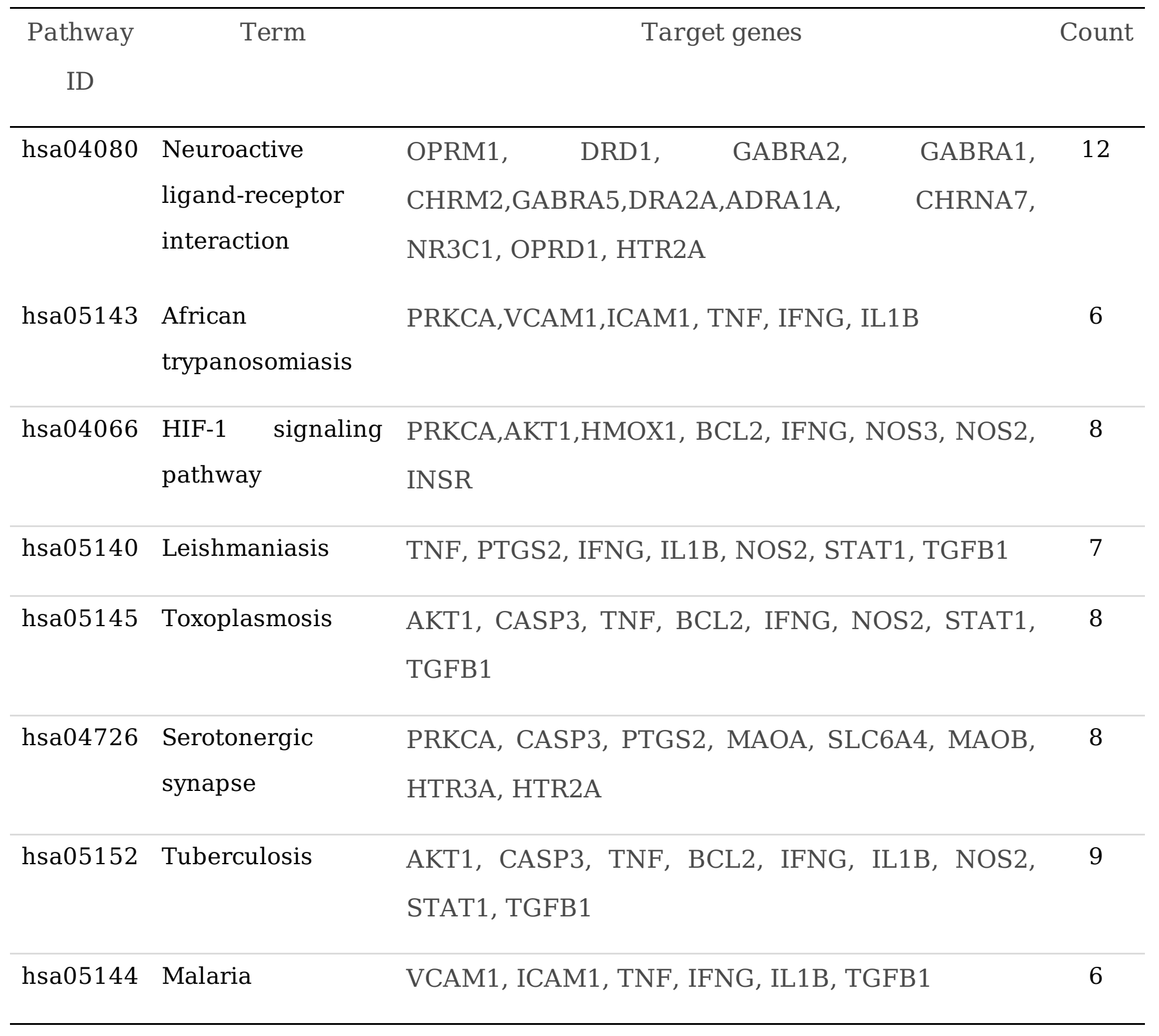

\section{Figures}



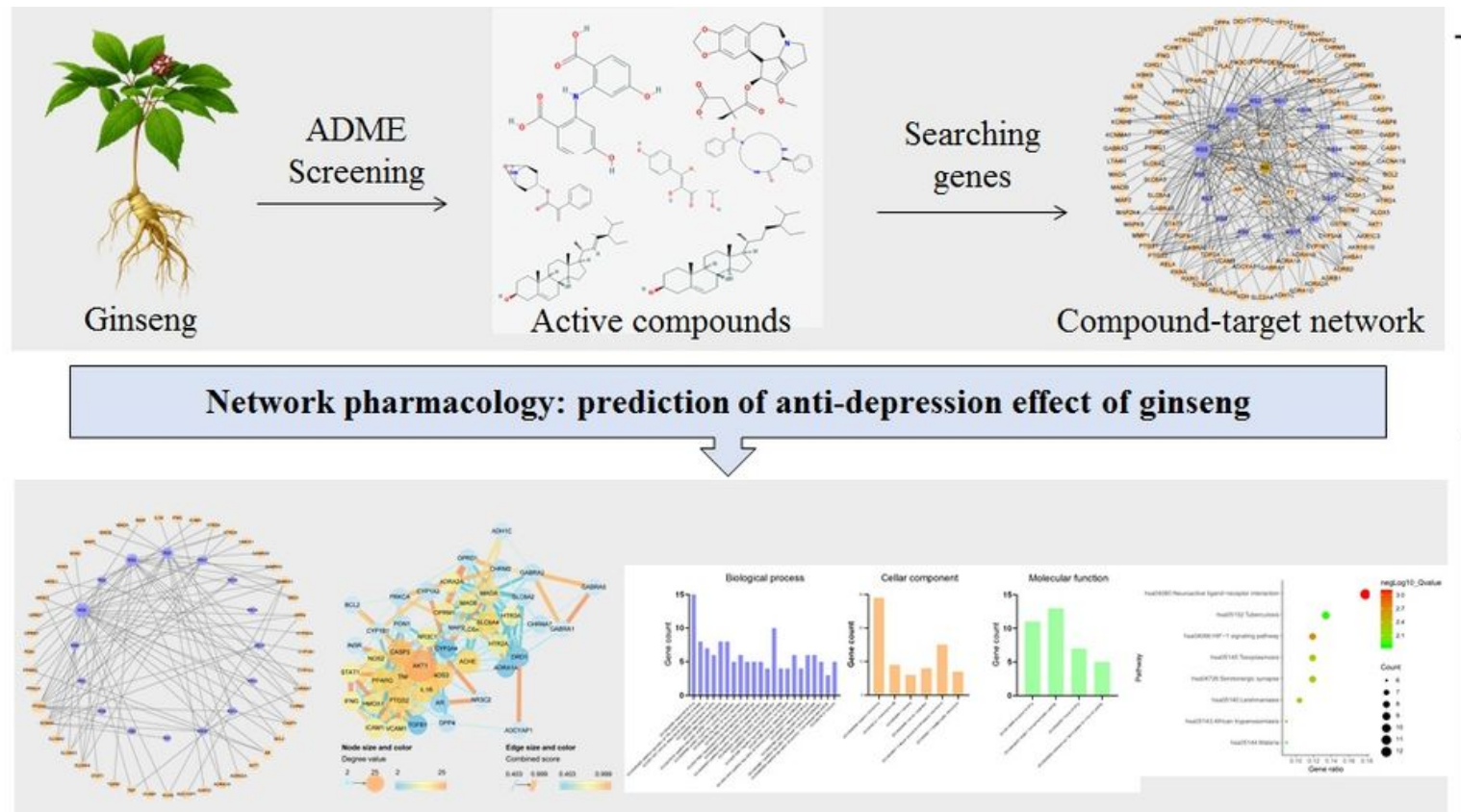

Compound-target-disease network

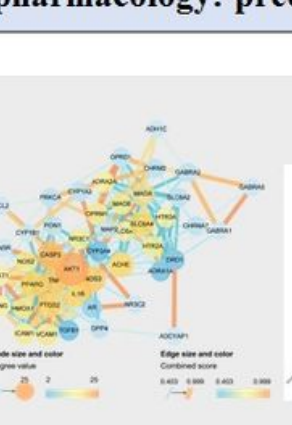

PPI network
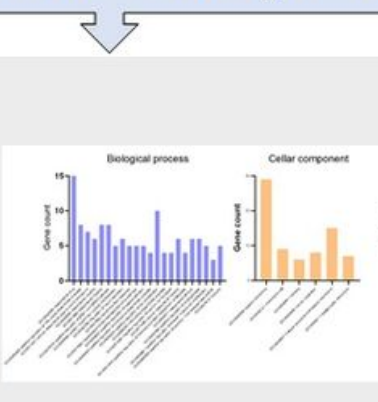
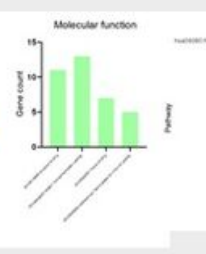

Main mechanism :

neurotransmitters

neurotrophic factors

neurogenesis

HPA axis

inflammatory response

\section{Figure 1}

The fowchart of the network pharmacology-based strategy for deciphering the mechanisms of ginseng on depression.

Fig.1 


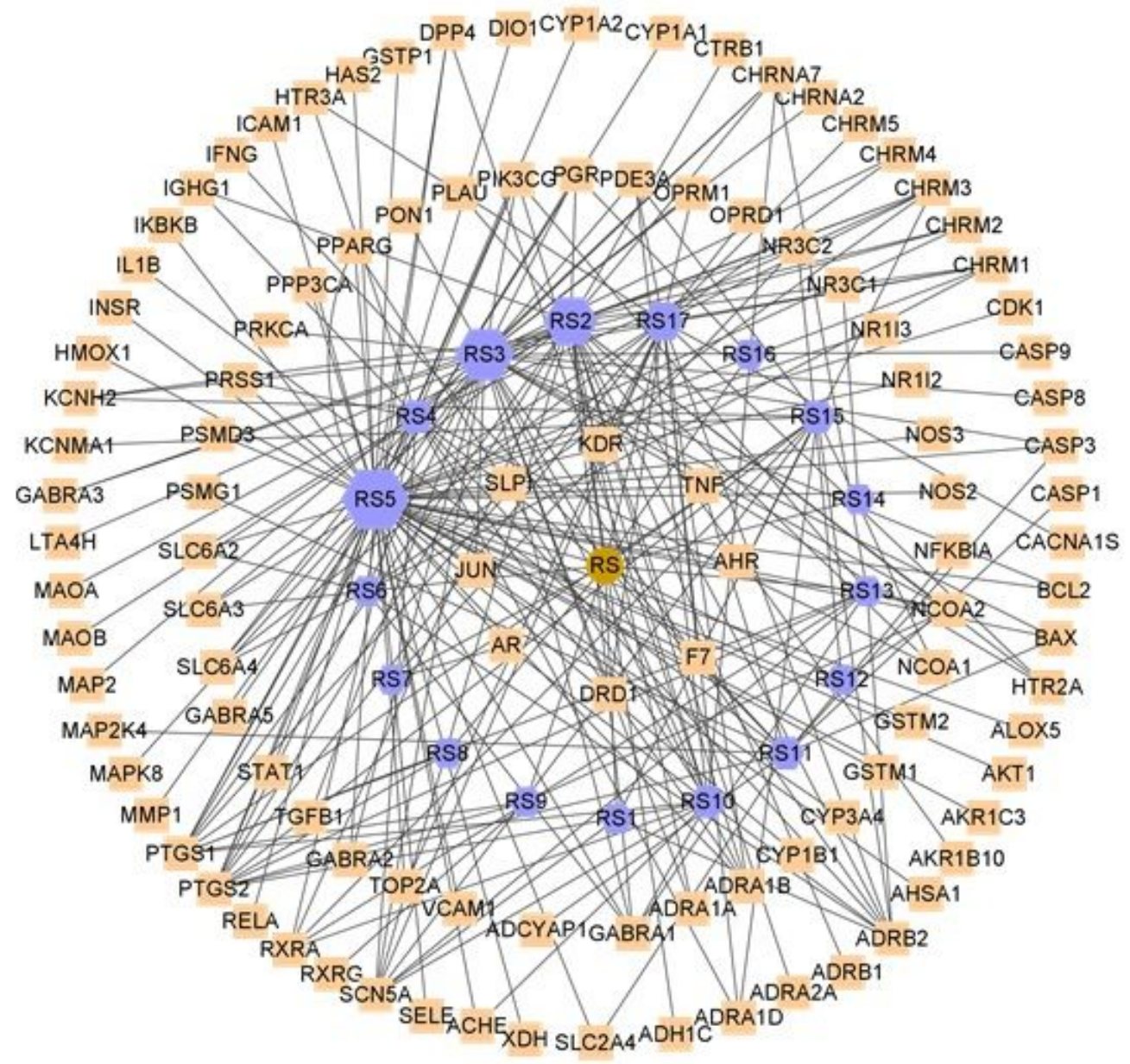

Figure 2

Compound-compound target network. The size of the node is proportional to the value of the degree. 
(a)

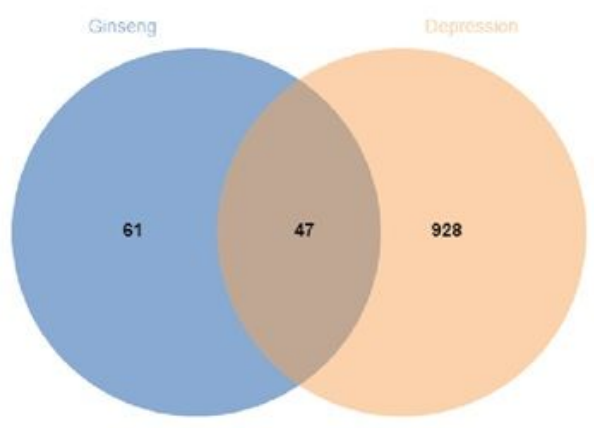

(b)

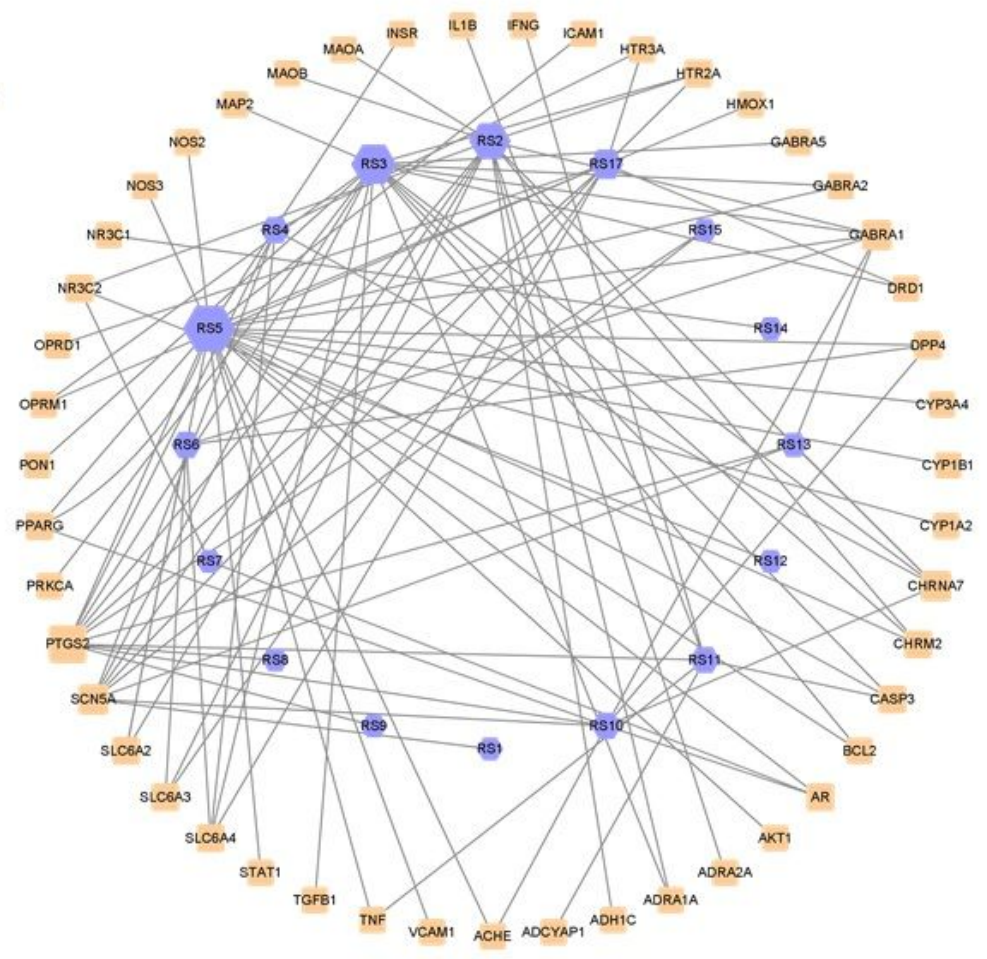

Fig. 3

\section{Figure 3}

(a)Venn diagram. (b)Compound-target-disease network with 63 nodes and 102 edges linking 16 compounds and 47 target genes related to depression. 


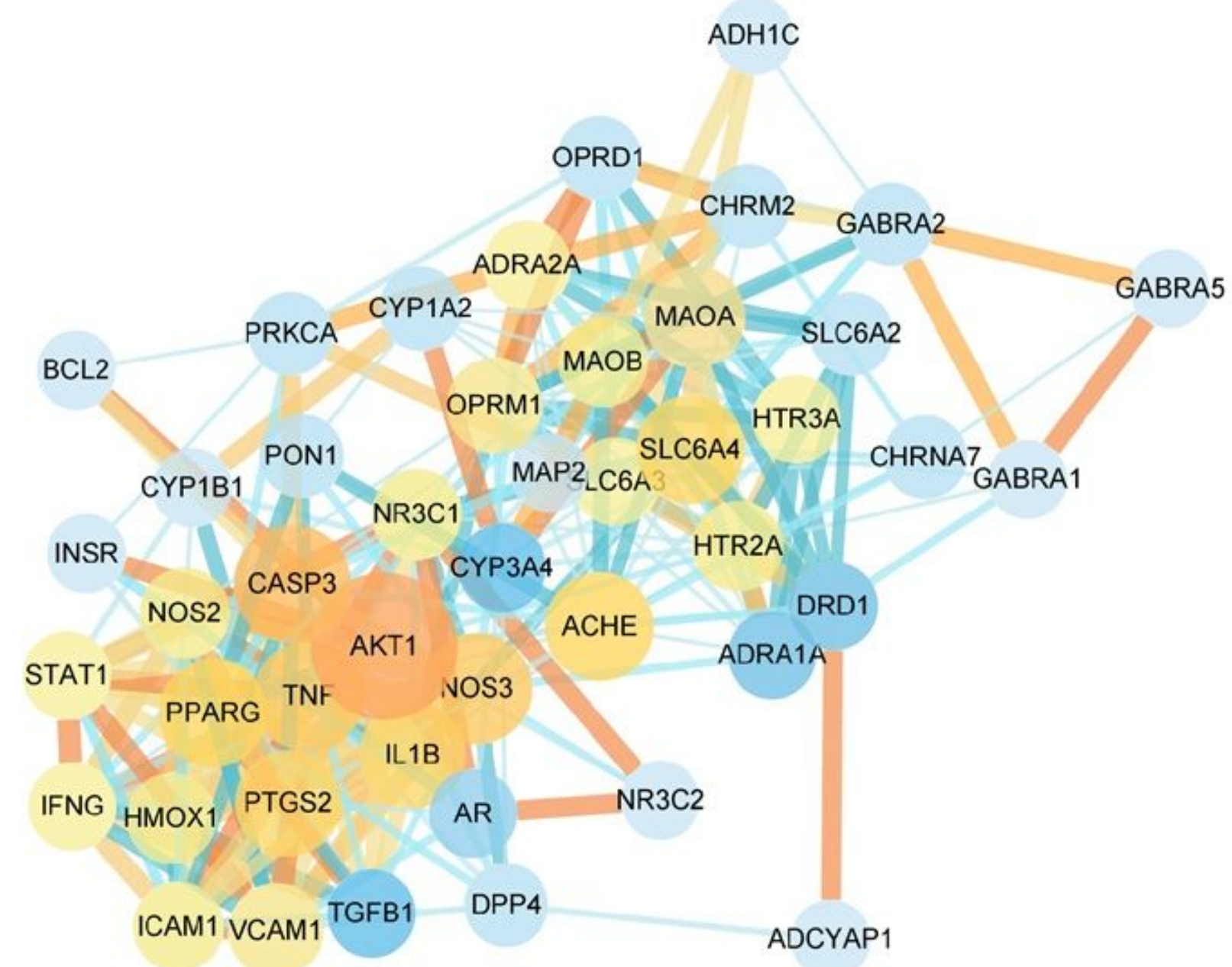

Node size and color

Degree value

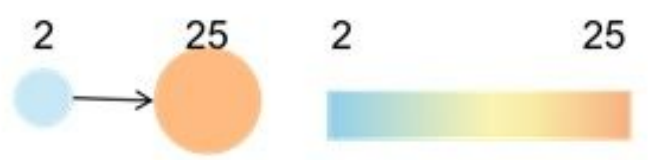

Edge size and color

Combined score

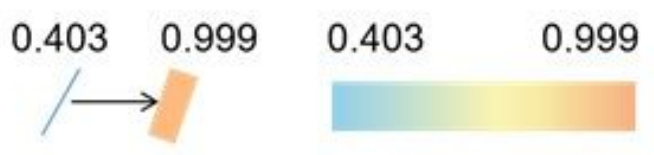

Fig.4

Figure 4

PPI network of depression-related targets in ginseng. 

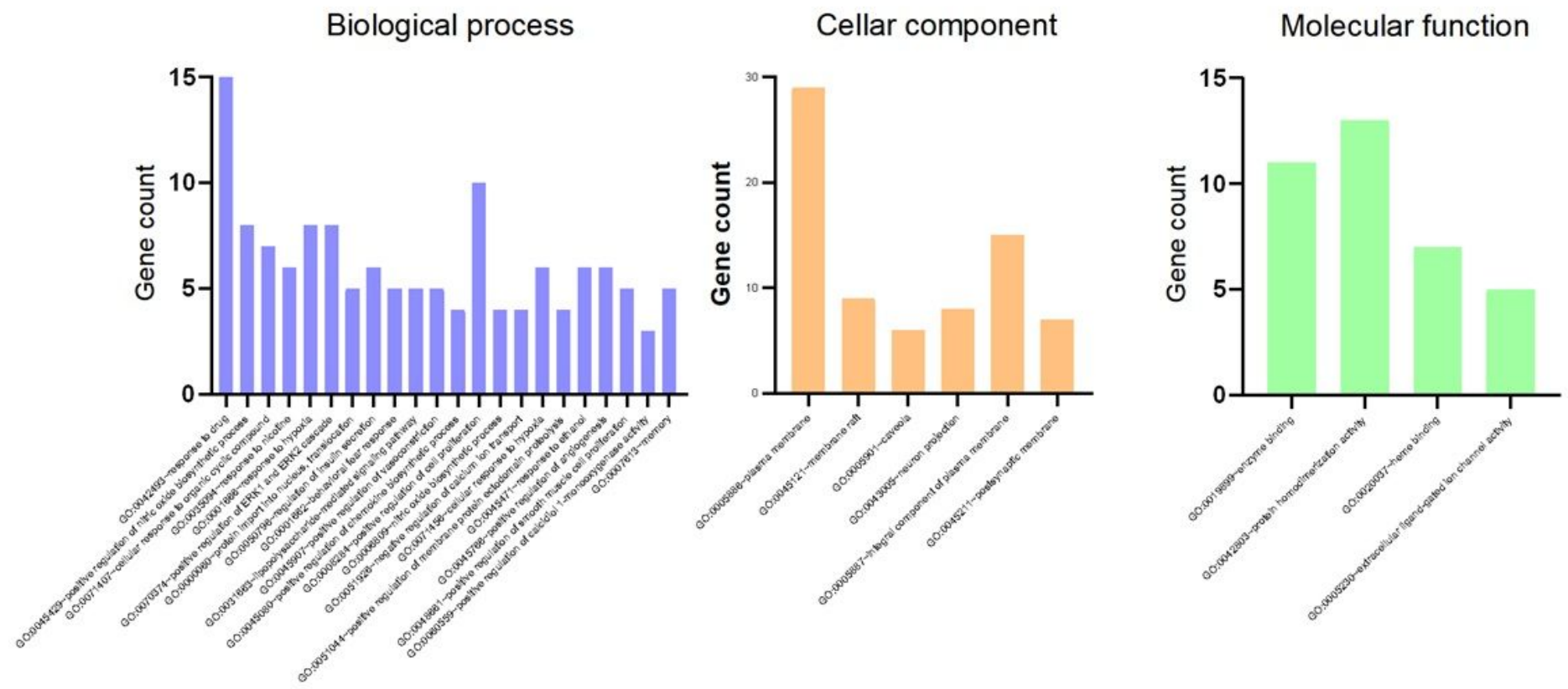

Fig.5

Figure 5

GO functional annotation analysis of 47 target genes were sorted according to FDR $<0.05$. 


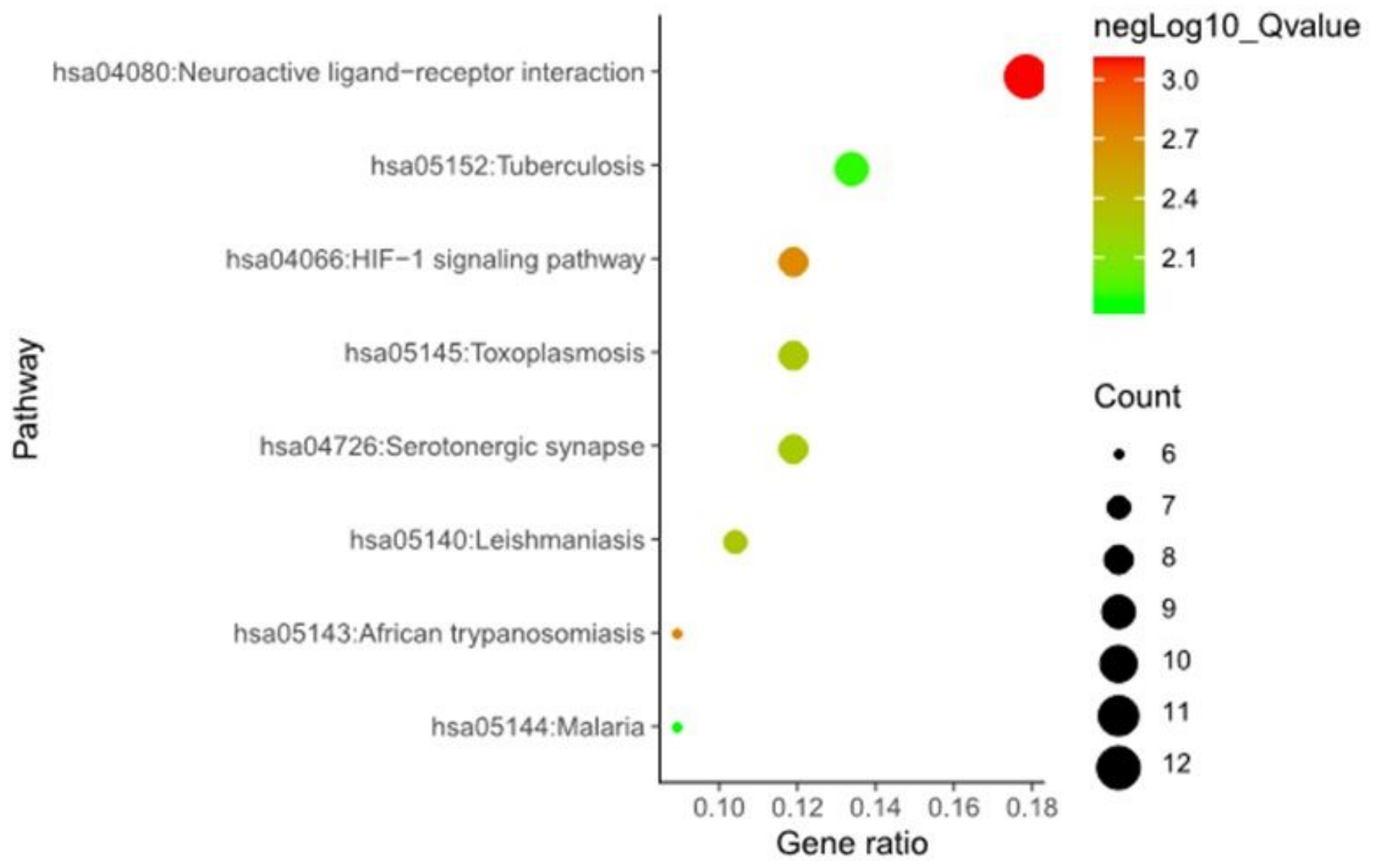

Fig.6

Figure 6

KEGG pathways analysis of 47 target genes were sorted according to $\mathrm{P}<0.01$ and $\mathrm{FDR}<0.05$. 